\title{
Changes in Head and Neck Oncologic Practice During the COVID-19 Pandemic
}

\author{
Robert M. Brody, MD ${ }^{1}$ \\ ${ }^{1}$ University of Pennsylvania
}

April 28, 2020

\begin{abstract}
Introduction: The COVID-19 pandemic has raised controversies regarding safe and effective care of head and neck cancer patients. It is unknown how much the pandemic has changed surgeon practice. Methods: A questionnaire was distributed to head and neck surgeons assessing opinions related to treatment and concerns for the safety of patients, self, family, and staff. Results: A total of 67 head and neck surgeons responded during the study period. Surgeons continued to recommend primary surgical treatment for oral cavity cancers. Respondents were more likely to consider non-surgical therapy for patients with early glottic cancers and HPV-mediated oropharynx cancer. Surgeons were least likely to be concerned for their own health and safety and had the greatest concern for their resident trainees. Conclusions: This study highlights differences in the willingness of head and neck surgeons to delay surgery or alter plans during times when hospital resources are scarce and risk is high.
\end{abstract}

Robert M. Brody, $\mathrm{MD}^{1}$, W. Greer Albergotti, $\mathrm{MD}^{2}$, David Shimunov, BA ${ }^{1}$, Elizabeth Nicolli, $\mathrm{MD}^{3}$, Brianna N. Harris, $\mathrm{MD}^{4}$, Andrés M. Bur, $\mathrm{MD}^{5}$

1. Department of Otorhinolaryngology - Head \& Neck Surgery, University of Pennsylvania, Philadelphia, PA

2. Department of Otolaryngology - Head \& Neck Surgery, Medical College of Georgia / Augusta University, Augusta GA

3. Department of Otolaryngology- Head \& Neck Surgery, University of Miami, Miami, FL

4. Department of Otolaryngology- Head \& Neck Surgery, Scripps Memorial, San Diego, CA

5. Department of Otolaryngology- Head \& Neck Surgery, University of Kansas, Kansas City, KS

\section{Corresponding Author}

Robert M. Brody, MD

Department of Otorhinolaryngology - Head \& Neck Surgery, University of Pennsylvania

3400 Spruce Street, 5 Ravdin

Philadelphia, PA, 19104

E-mail: Robert.brody2@pennmedicine.upenn.edu

Disclosures

This work has never been published or presented anywhere. The authors have no financial or industry relationships to disclose. There are no sources of funding to disclose.

Running Title 
Survey of Head \& Neck Surgeon Preferences and Concerns During COVID-19 Pandemic

Keywords

SARS-CoV-2, COVID-19, Coronavirus, Head and Neck Cancer, Treatment Delays, Wellness

Abstract

Introduction: The COVID-19 pandemic has raised controversies regarding safe and effective care of head and neck cancer patients. It is unknown how much the pandemic has changed surgeon practice.

Methods: A questionnaire was distributed to head and neck surgeons assessing opinions related to treatment and concerns for the safety of patients, self, family, and staff.

Results: A total of 67 head and neck surgeons responded during the study period. Surgeons continued to recommend primary surgical treatment for oral cavity cancers. Respondents were more likely to consider non-surgical therapy for patients with early glottic cancers and HPV-mediated oropharynx cancer. Surgeons were least likely to be concerned for their own health and safety and had the greatest concern for their resident trainees.

Conclusions: This study highlights differences in the willingness of head and neck surgeons to delay surgery or alter plans during times when hospital resources are scarce and risk is high.

Introduction

The coronavirus disease 19 (COVID-19) caused by the severe acute respiratory syndrome coronavirus 2 (SARS-CoV-2) was declared a pandemic by World Health Organization (WHO) on March 11, 2020. ${ }^{1}$ This pandemic has placed increased stress on health care systems, both due to critical resource shortages and delays in care. Health care providers now must find new ways of safely providing cancer care without compromising patient outcomes, or overutilizing precious resources. ${ }^{2}$ The American Academy of Otolaryngology - Head \& Neck Surgery has recommended surgeons delay elective cases to allow for improved safety of staff and patients, preservation of critical personal protective equipment (PPE), and to expand hospital capacity during the COVID-19 pandemic. ${ }^{3}$ Additionally, the American College of Surgeons (ACS) has provided guidance defining the acuity of cancer surgeries, with most mucosal cancers of the upper aerodigestive tract meeting the criteria for Tier 3a (high acuity surgery/healthy patient). Accordingly, delays in treatment should not occur. ${ }^{4}$ The ACS feels that "the risk to the patient should include an aggregate assessment of the real risk of proceeding and the real risk of delay, including the expectation that a delay of 6-8 weeks or more may be required to emerge from an environment in which COVID-19 is less prevalent". ${ }^{4}$

Head and neck cancer care poses a unique challenge during the COVID-19 pandemic because increasing evidence has emerged that Otolaryngologists are at significantly higher risk of infection due to exposure to the upper airway and respiratory droplets. ${ }^{5,6}$ Additionally, reasonable alternative treatment options are lacking for many head and neck malignancies and treatment delay leads to increased morbidity and mortality. ${ }^{7,8}$ Head and neck cancer is unique in that oral cavity cancers, human papillomavirus (HPV)-negative oropharyngeal squamous cell carcinoma (OPSCC), and advanced laryngeal cancers are considered aggressive malignancies and there is no widely-accepted neoadjuvant treatment option that can allow definitive surgery to be delayed. Other subtypes such as HPV-mediated OPSCC and early laryngeal squamous cell carcinoma can be treated either with radiation-based or surgical-based therapy, which offer equivalent oncologic outcomes.

Despite ACS recommendations that delays in cancer treatment be avoided during the COVID-19 pandemic, treatment delays are anticipated and are currently occurring. This can be expected to alter the management strategies as head and neck surgeons and radiation oncologists weigh the risks and benefits of viral exposure both to the patient and to operating room and clinic staff. Furthermore, patients who unknowingly have surgery during the incubation period of COVID-19 infection appear to be at extremely high risk of morbidity and may have approximately $20 \%$ risk of mortality ${ }^{8}$. There is also a high level of concern over the risk of immunosuppression with systemic chemotherapy during the ongoing pandemic. These risks must be balanced with the risk of cancer progression by delaying therapy as well as the psychological impact on patients of 
delayed or altered plans of care. ${ }^{10-12}$ It has been established in the literature that increases in the duration of time between diagnosis and definitive therapy is associated with decreased overall and disease-free survival. ${ }^{7,8}$ In addition, delays in surgery may result in increased morbidity due to disease progression necessitating more extensive tumor resection.

Because of the potential increase in morbidity and mortality, most head and neck surgeons including the authors advocate for initiation of treatment within one month of the patient's presentation to clinic. However, in the COVID-19 era it is unclear what the appropriate length of time from evaluation to initiation of treatment should be given limited resources and new concern over potential morbidity and mortality from early initiation of treatment.

Therefore, the primary objective of this study was to assess head and neck surgeons' priorities and the extent to which they would consider delaying surgery or changing treatment modality in the era of COVID-19. This knowledge may help guide the development of guidelines and support treatment decisions both now and during future pandemics. The secondary objective was to understand surgeons' concerns regarding safety of treatment for their patients during the COVID-19 pandemic as well as the safety of their families, their staff, and themselves.

Methods

This descriptive study was performed after a waiver was obtained from internal review board at the University of Pennsylvania. A questionnaire assessing three separate domains was conducted. Demographics included surgeon age, academic versus private practice, and zipcode in which the surgeon practices. Utilizing zipcode data, participants were subsequently grouped into two categories: those practicing in an area with less than 25,000 confirmed cases of COVID-19 vand those practicing in an area with greater than or equal to 25,000 confirmed cases. These domains included surgeon preferences related to treatment delays and referral for nonsurgical therapies; concerns regarding various treatment-related complications secondary to COVID-19; and worry about the health and safety of self, family, staff, and trainees. Questions related to treatment delays and nonsurgical therapy specifically addressed management related to early tumor stage oral cavity cancer, locally advanced oral cavity cancer, HPV-associated oropharyngeal cancer, early tumor stage glottic cancer, locally advanced laryngeal cancer, and recurrent laryngeal cancer requiring salvage surgery.

The survey was administered through the Research Electronic Data Capture (REDCap) ${ }^{13}$ platform and was distributed amongst head and neck surgeons in different geographic regions across the United States and Canada using message boards and social media. Questions regarding respondents' demographic information included age, zipcode in which they practice, and whether they practice in an academic or private hospital. The survey was completed by 67 respondents over a 5 day period (April 9 - April 14, 2020). Statistical analyses were performed using STATA/IC 15.0 software (Stata Corporation, College Station, TX).

\section{Results}

A total of 67 head and neck surgeons responded to the survey during the study period. Fifty-nine (88.1\%) practiced in an academic setting while the remaining $8(11.9 \%)$ worked in a private practice setting. The majority of respondents were between the ages of 30 and $39(52.2 \%$; Table 1$)$. There were no differences in responses when stratifying by age or whether the participants were practicing in an area with a high prevalence of COVID-19.

\section{Primary surgical versus primary nonsurgical therapy}

For oral cavity cancers, the majority of physicians would not change their current practice and still favored primary surgical therapy (82.1\% early T-stage and $79.1 \%$ locally advanced) . A small percentage of respondents $(7.5 \%$ early T-stage, $10.4 \%$ locally advanced) stated that they would be more likely to refer for (chemo)radiotherapy but no respondents were very likely to change their normal practice (Figure 1). A majority of respondents were more likely to consider non-surgical therapy for patients with early glottic cancers (62.1\%) and HPV-mediated oropharynx cancer (68.7\%) during the COVID-19 pandemic as compared to 
their normal practice. For locally advanced T4a laryngeal cancers, a minority of respondents (25.7\%) were willing to deviate from the standard of care to offer non-surgical therapy.

\section{Willingness to delay treatment during the COVID-19 surge}

The majority of respondents were willing to delay their normal treatment timeline by 2 weeks or 4 weeks for early tumor stage oral cavity cancer $(53.7 \%)$, while few were willing to delay 6 weeks $(10.4 \%)$ and no respondents were willing to delay beyond 6 weeks (Figure 2). Respondents were least likely to delay treatment for a locally advanced oral cavity cancer as compared to other tumors that were assessed. For early stage glottic cancers and HPV-mediated oropharynx cancers, the largest percentage of surgeons were willing to wait up to 4 weeks with a minority of respondents willing to wait up to 8 weeks. Participants responded similarly in regards to locally advanced laryngeal cancers and recurrent laryngeal cancers with the largest group willing to wait up to 4 weeks for surgical therapy.

\section{Concerns related to complications from COVID-19 during treatment}

Head and neck surgeons were most concerned about complications secondary to COVID-19 infection in patients who were receiving chemotherapy (Figure 3).

Concerns related to the well-being of self, staff, trainees, and family during the COVID-19 pandemic

Notably, head and neck surgeons were the least likely to be concerned for their own health and safety as compared to that of their residents, family, or staff. Surgeons had the greatest concern for the wellbeing of their resident trainees during the COVID-19 pandemic. The majority of respondents expressed moderate concern for the staff and their family during the COVID-19 pandemic (Figure 4).

\section{Discussion}

As the COVID-19 pandemic has forced head and neck surgeons to triage which patients should be operated urgently versus which can be delayed indefinitely, this study helps to provide granular data regarding the opinions of 67 head and neck surgeons across multiple geographic locations within the United States and Canada.

Our respondents still feel it is necessary to operate on patients with cancers of the larynx, oropharynx, and oral cavity in setting of the COVID-19 pandemic, however there are notable differences between sites and stages. As oral cavity squamous cell carcinoma is regarded as a primarily surgical disease due to higher treatment related complications and poorer outcomes with primary radiotherapy, our respondents were unwilling to refer patients for nonsurgical therapy. This trend is similarly noted in the setting of a recurrent laryngeal cancer as no other curative treatment is available. It was interesting to note that a minority of respondents were willing to refer a locally advanced T4a laryngeal cancer for primary nonsurgical treatment. While this is not standard of care, it may reflect the hope that a patient may respond to radiation therapy and undergo salvage at a later date if there is only a partial response or if the patient has a nonfunctional larynx.

Most respondents were willing to deviate from their standard practice and recommend primary (chemo)radiotherapy for early laryngeal cancers and HPV-mediated oropharynx cancers. While nonsurgical therapy is a standard of care for these cancers, the fact that surgeons are more willing to recommend them during the COVID-19 pandemic as compared to their normal practice indicates that our respondents felt that it might be safer for the patient and for the healthcare team if surgical treatment was deferred.

Although surgeons may wish to shunt patients towards radiation oncology in the hopes of limiting resource utilization and exposure in the peri-operative setting, the burden placed on radiation oncologists must also be considered. With 6 to 7 weeks of treatment, there is a substantial amount of travel and exposure to health care staff during treatment. The risk of treatment delays or breaks must also be taken into account if the patient becomes infected or if enough staff are quarantined resulting in a reduced capacity to treat patients.

Most surgeons were not willing to delay treatment beyond 4-6 weeks during the COVID-19 pandemic. This 
is likely related to the known risk morbidity and mortality among patients with head and neck cancer that results from treatment delays. ${ }^{7,8}$ In a systematic review of 51 studies, Schutte et al found that treatment delays led to reduced overall survival and disease specific survival, as well worsened functional and psychosocial outcomes. They recommended timely treatment to improve outcomes in HNSCC. ${ }^{7}$ Similarly, in 956 patients, Liao et al found that delays in treatment led to lower overall survival and higher risk of recurrence. ${ }^{8}$ This highlights the importance of timely intervention, even in the era of COVID-19, to maintain a high level of care. The need for timely intervention must be weighed with opposing concerns that necessitate delays or alterations in treatment paradigms to protect health care workers due to a lack of PPE or availability of preoperative COVID-19 testing. ${ }^{6}$

Additionally, there are significant concerns related to complications from COVID-19 during treatment that need to be taken into consideration. If patients are at high risk of mortality with SARS-CoV-2 infection due to age or comorbidities, then it is prudent to delay surgery, especially at the height of the pandemic. As demonstrated by the data presented above, surgeons' willingness to delay surgery may vary. Increased risk of complications in the setting of SARS-CoV-2 infection may result from respiratory compromise due to aspiration pneumonia, atelectasis, or pneumonitis after airway surgery. Patients undergoing radiation may also have a theoretical risk of developing a more severe form of COVID-19 in the setting of concurrent mucositis, aspiration pneumonitis, or weight loss during radiation. Lastly, the immunocompromised state induced by chemotherapy may also increase the risks of respiratory compromise and failure in the setting of a COVID-19 infection.

Lastly, our respondents' answers regarding their own health as well as the health of their family, staff, and trainees are heartening. The head and neck surgeons responding to this survey must limit exposure to their families while continuing to function as essential workers for their patients on the front lines of this pandemic. Our respondents greatest concern is related to the risk that is placed upon their staff and more so on their residents. Although resident duty hours have been substantially decreased amidst the COVID-19 pandemic to limit exposure, otolaryngology residents in academic centers still perform invaluable emergency services and provide assistance in complex cases that require more than a single surgeon. Shielding our residents from harm during this pandemic is of great concern as demonstrated by the findings of this study. Lastly, head and neck surgeons seem to have the least concern for their own health and safety during the pandemic. While this is laudable, it is important to stress physical and mental safety amongst our colleagues during this health care crisis.

\section{Conclusion}

This study demonstrates the range of variability amongst head and neck surgeons in their willingness to delay cases during times when hospital resources are scarce and risk is high. This work will help to triage certain cases and ensure that other cases are not inappropriately delayed beyond a reasonable timeframe.

\section{References}

1. https://www.jwatch.org/fw116441/2020/03/11/who-declares-covid-19-pandemic

2. Emanuel EJ, Persad G, Upshur R, Thome B, Parker M, Glickman A, Zhang C, Boyle C, Smith M, Phillips JP. Fair Allocation of Scarce Medical Resources in the Time of Covid-19. N Engl J Med. 2020 (Epub).

3. Academy of Otolaryngology-Head and Neck Surgery. New Recommendations Regarding Urgent and Nonurgent Patient Care. https://www.entnet.org/content/new-recommendations-regarding-urgent-andnonurgent-patient-care (Accessed 15 April 2020).

4. COVID-19: Guidance for Triage of Non-Emergent Surgical Procedures. https://www.facs.org/covid19/clinical-guidance/triage. Accessed March 29, 2020. 
5. Shuman A and Campbell B. An ethical framework for head and neck cancer care impacted by COVID19. Otolaryngology- Head and Neck Surgery, 2020. E-publication AHNS COVID-19 Bulletin Board

6. Givi B, Schiff BA, Chinn SB et al. Safety Recommendations for evaluation and surgery of the head and neck during the COVID-19 pandemic. JAMA Otolaryngol- Head Neck Surg 2020; epub ahead of print.

7. Schutte HW, Heutink F, Wellenstein DJ et al. Impact of time to diagnosis and treatment in head and neck cancer: a systematic review. Otolaryngology Head and Neck Surgery, 2020; 162(4) 446- 457.

8. Liao DZ, Schlecht NF, Rosenblatt G, et al. Association of delayed time to treatment initiation with overall survival and recurrence among patients with head and neck squamous cell carcinoma in an underserved urban population. JAMA Otolaryngol Head Neck Surg 2019; Epub

9. Lei, S., Jiang, F., Su, W., Chen, C., Chen, J., Mei, W., Zhan, L., Jia, Y., Zhang, L., Liu, D., Xia, Z., Xia, Z. (2020).Clinical characteristics and outcomes of patients undergoing surgeries during the incubation period of COVID-19 infectionEClinicalMedicine

10. Werner MT, Carey RM, Albergotti WG, Lukens JN, Brody RM. Impact of the COVID-19 Pandemic on the Management of Head and Neck Malignancies. Otolaryngol Head Neck Surg 2020 (Epub).

11. Balakrishnan K, Schechtman S, Hogikyan ND, Teoh AYB, McGrath B, Brenner MJ. COVID-19 Pandemic: What every Otolaryngologist - Head \& Neck Surgeon Needs to Know for Safe Airway Management. Otolaryngol Head Neck Surg 2020 (Epub).

12. Liang W, Guan W, Chen R, et al. Cancer patients in SARS-CoV-2 infection: a 133 nationwide analysis in China. Lancet Oncol. 2020;21(3):335-337.

13. Harris PA, Taylor R, Thielke R, Payne J, Gonzalez N, Conde JG. Research electronic data capture (REDCap)-a metadata-driven methodology and workflow process for providing translational research informatics support. J Biomed Inform 2009; 42:377-381

14.

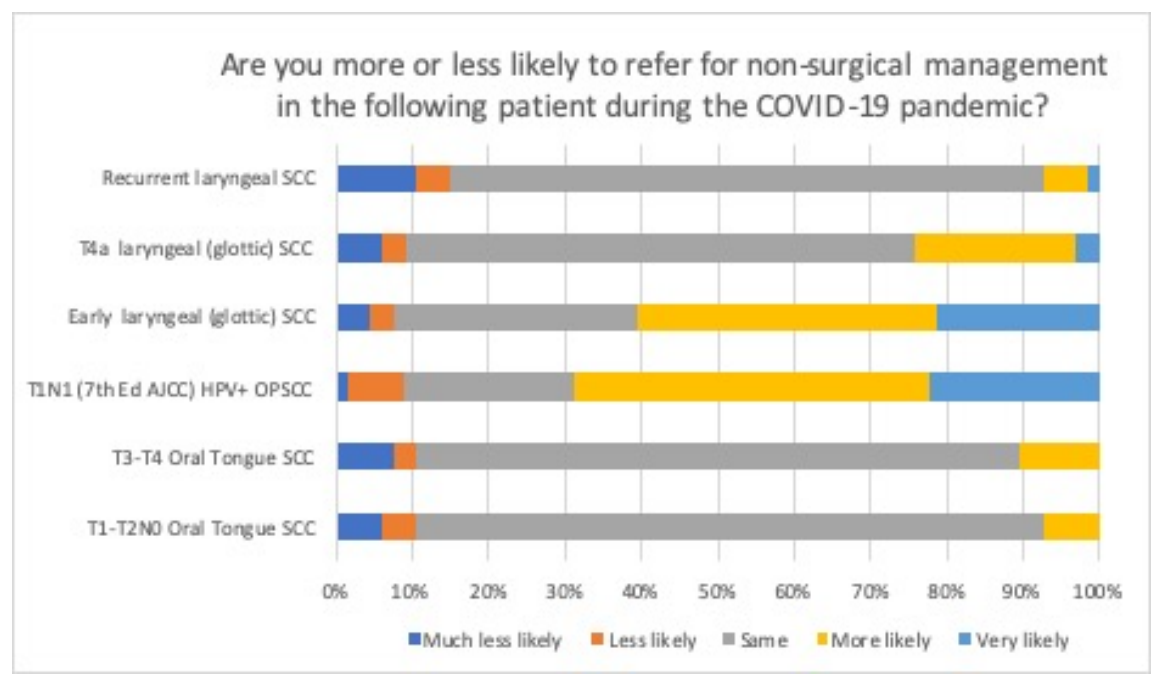

Figure 1: This is a caption 


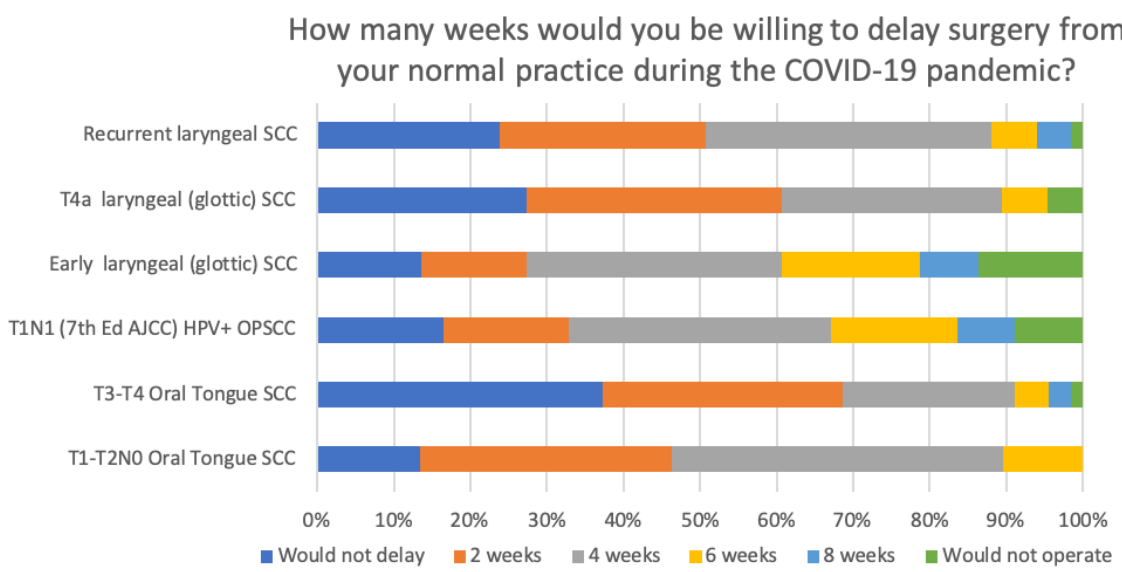

Figure 2: This is a caption

\section{Concerns regarding complications during treatment}

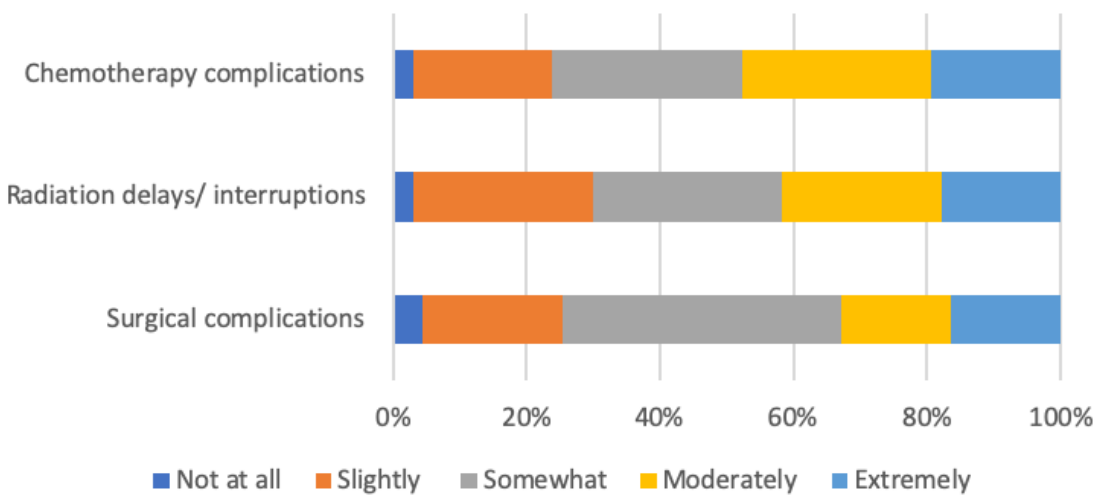

Figure 3: This is a caption 


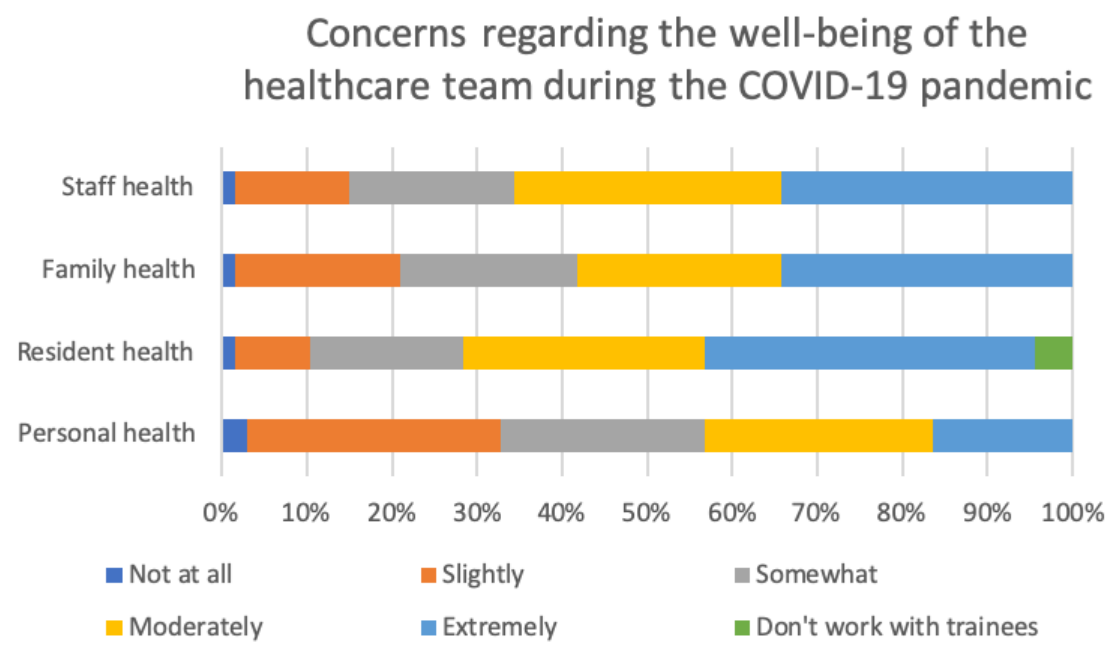

Figure 4: This is a caption

\section{Hosted file}

Table_1 (1).docx available at https://authorea.com/users/312328/articles/445013-changes-in-headand-neck-oncologic-practice-during-the-covid-19-pandemic 